\title{
Message from the President
}

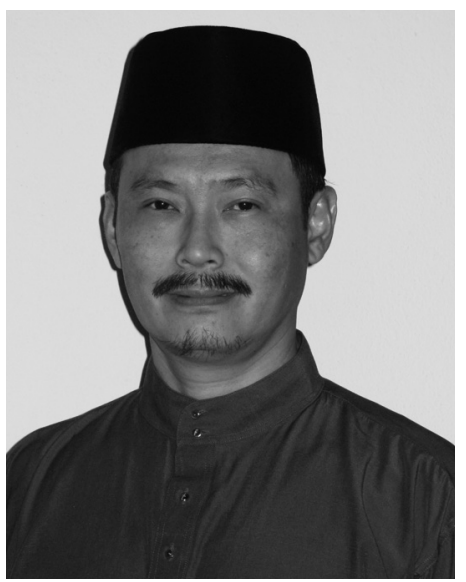

\section{Dear Readers,}

The publications of this journal is one of the many activities undertaken by the Malaysian Institute of Planners (MIP) to propagate knowledge and information pertaining to town and country planning to its members as well as the public. This Journal also acts as a medium for MIP members and others to engage in research and writing articles that could contribute to the advancement of the theory and practice of town and country planning. Published articles in this Journal means for MIP members, is fulfilling their CPD point requirement. As to the academic contributors, journal's indexed in SCOPUS will be very meaningful as it adds extra point in terms of their involvement in research and publication.

This year, MIP's journal has moved extra mile by producing a special issue dedicated specifically on Langkawi Geopark. Ten related titles researched and written by a group of experts from LESTARI, UKM and LADA staff had contributed in this special issue. Topics which are covered and discussed in this issue would definitely promote better understanding on current issues relating to our first geopark, not only in Malaysia, but South East Asia dated back about 550 million years ago as endorsed by UNESCO in 2007. The articles discussed rigorously not only on geopark concept that made up of more than mere geological structures and landscape but also about how the local communities within it can sustain and nurture this geological heritage through effective conservation efforts and promotion of ecotourism. Experiences and suggestions put forward by the authors in this Journal could be used or adopted into practice by MIP members and authorities in carrying out their professional role in maintaining our very own world heritage. Congratulation to the authors for their excellent effort and materials published in this special issue.

On behalf of the council I would like to thank the editors. I would like to urge members of MIP and others to make full use of this Journal.

\section{Prof. Dato' Dr. Alias Abdullah \\ PRESIDENT}

(2011-2013) 